PROCEEDINGS OF THE

AMERICAN MATHEMATICAL SOCIETY

Volume 129, Number 5, Pages 1493-1498

S 0002-9939(01)05554-X

Article electronically published on January 8, 2001

\title{
GLUING COPIES OF A 3-DIMENSIONAL POLYHEDRON TO OBTAIN A CLOSED NONPOSITIVELY CURVED PSEUDOMANIFOLD
}

\author{
D. BURAGO, S. FERLEGER, B. KLEINER, AND A. KONONENKO \\ (Communicated by Christopher Croke)
}

\begin{abstract}
Let $S$ be a smooth 3-dimensional nonpositively curved Riemannian manifold with corners, whose boundary consists of a finite number of geodesically convex nonpositively curved faces (for example, a Euclidean or hyperbolic polyhedron). We show that it is always possible to glue together finitely many copies of $S$ so as to get a nonpositively curved pseudomanifold without boundary.
\end{abstract}

\section{INTRODUCTION}

In this paper we will study the following geometric problem. Let $P$ be a finite polyhedron, or more generally a smooth compact nonpositively curved Riemannian manifold with corners so that every codimension 1 face is geodesically convex. We ask whether it is possible to take finitely many copies of $P$ and identify their codimension 1 faces in pairs so as to obtain a closed pseudomanifold 1 (see $\mathrm{Sp}$ p. 148] for the definition) with nonpositive curvature in the sense of A. D. Alexandrov. It is easy to do this when $P$ has dimension 2 , but when $\operatorname{Dim}(P) \geq 3$ the problem is much more subtle. In fact, it seems doubtful that it can be done for all $P$ of dimension $\geq 4$. In this paper we will solve the 3 -dimensional case of this gluing problem using Thurston's geometrization theorem for Haken manifolds; see Theorem 1 below.

Our interest in this problem originated in constructions of BFK1, BFK2, BFK3. which established a natural connection between semi-dispersing billiard systems and the geometry of certain spaces arising from a gluing procedure similar to the one described above. For instance, one can reduce the proof of the uniform boundedness of the number of collisions in a system of several hard balls colliding elastically in empty Euclidean or hyperbolic space to verifying that auxiliary spaces built from finitely many isometric copies of an associated billiard table have nonpositive curvature. The first step of this reduction is to "develop" billiard trajectories

Received by the editors August 31, 1998.

2000 Mathematics Subject Classification. Primary 51K10, 53C20; Secondary 52B10.

The first author was partially supported by a Sloan Foundation Fellowship and NSF grant DMS-9803129. The second author was partially supported by a Sloan Dissertation Fellowship. The third author was supported by a Sloan Foundation Fellowship, and NSF grants DMS-9505175, DMS-96-26911. The fourth author was supported by NSF grant DMS-9803092.

${ }^{1} \mathrm{~A}$ related easier task would be to glue together copies of $P$ to get a compact geodesically complete nonpositively curved space. 
as geodesics in an auxiliary space. A nagging drawback of the constructions in BFK1, BFK2, BFK3 is that the auxiliary space depends on the combinatorial class of the chosen trajectory (i.e., the sequence of "collisions" encountered by the trajectory). In BFK3] we asked whether there is a "global model space" for every billiard system - an Alexandrov space in which one can develop all billiard trajectories, regardless of combinatorial class. The existence of a global model space for a billiard system implies that it is a finite quotient of the geodesic flow of an Alexandrov space (see BFK3] for a detailed discussion).

Another reason to consider this gluing problem is that it might provide a rich supply of easily studied examples.

Our main theorem is

Theorem 1. Let $S$ be a 3-dimensional smooth compact nonpositively curved Riemannian manifold with corners, such that all the corners are nondegenerate (i.e., all the one and two-dimensional angles in the corners are nonzero), and the boundary of $S$ consists of a finite number of geodesically convex nonpositively curved 2 faces $F_{i}, i=1, \ldots, n$. Then it is possible to glue together finitely many copies $S_{j}$, $j=1, \ldots, k$, of $S$ so that

1. The $S_{j}$ 's are glued by identifying their faces isometrically in pairs.

2. The pseudomanifold $M=\bigcup_{j=1, \ldots, k} S_{j}$ has nonpositive curvature.

We remark that the related problem of gluing together copies of a polyhedron to obtain a nonpositively curved topological manifold is also interesting 3 Polyhedra which admit such gluings satisfy very restrictive conditions. For instance, one can easily see that a 3 -dimensional nonpositively curved pseudomanifold produced by gluing copies of a regular (constant curvature) tetrahedron will always have negative Euler characteristic, and hence such a space is never a manifold.

\section{Proof of Theorem 1}

We will present the proof of Theorem 1 when $S$ is a three-dimensional Euclidean simplex. The proof in the general case follows exactly the same outline and the reader should have no difficulties reconstructing it from the case of a Euclidean simplex. We restrict ourselves to this case to avoid cumbersome notation and minor technicalities that would lengthen and obscure the exposition.

Let $S=V_{1} V_{2} V_{3} V_{4}$ be a 3 -dimensional Euclidean simplex. Without loss of generality we may assume that the shortest edge of $S$ has length 3. Denote by $F_{i}$ the face of $S$ opposite to the vertex $V_{i}$ of $S, i=1,2,3,4$.

2.1. Outline of the proof. We start by constructing, for every $K \in \mathbb{N}$, a polyhedral pseudo-manifold $M_{K}$ such that every edge of $M_{K}$ belongs to at least $K$ copies of $S$ (and, of course, $M_{K}$ is constructed out of a finite number of isometric copies of $S$ ). When $K$ is large enough $M_{K}$ will have nonpositive curvature everywhere except possibly at the vertices. To get nonpositive curvature at the vertices, we have to arrange that the vertex links are "large", i.e. that they have no closed geodesics of length $<2 \pi$. It turns out that if $K$ is sufficiently large, then the

\footnotetext{
${ }^{2}$ The faces must have nonpositive intrinsic curvature in order for $S$ to be an Alexandrov space with nonpositive curvature.

${ }^{3}$ Recently, we obtained a complete solution to the 3-dimensional case of this question. However, since our analysis requires a method different from one presented in this paper, we will discuss it elsewhere.
} 
shortest closed geodesic in a vertex link has to be homotopically nontrivial in the link. To complete the proof, we find a branched covering space $M \rightarrow M_{K}$ which branches at the vertices of $M_{K}$ (recall that the vertex links of $M_{K}$ are typically not simply connected); the vertex links of $M$ cover the vertex links of $M_{K}$, and by choosing the branched cover suitably (see below), we are able to eliminate short homotopically nontrivial closed geodesics in the vertex links.

To find branched covers of $M_{K}$, we (temporarily) remove small convex polyhedral neighborhoods $B_{n}, n=1, \ldots, m$, of the vertices of $M_{K}$ to get a space $M_{K}^{0}$. Finite covers of $M_{K}^{0}$ are in 1-1 correspondence with finite branched covers of $M_{K}$ with branch locus contained in the vertex set of $M_{K}$. In order to construct the branched cover $M \rightarrow M_{K}$ so that vertex links in $M$ have no short homotopically nontrivial geodesics, it is enough to find a finite index subgroup in $\pi_{1}\left(M_{K}^{0}\right)$ which does not contain the conjugacy classes of $\pi_{1}\left(M_{K}^{0}\right)$ corresponding to short closed geodesics in the vertex links of $M_{K}$. Finding this subgroup is the main difficulty of the proof. To overcome it we show that for large enough $K, \pi_{1}\left(M_{K}^{0}\right)$ is a linear group, and hence is residually finite $\mathrm{Ma}$.

When $K$ is large enough, $M_{K}^{0}$ has nonpositive curvature and its boundary components are nonpositively curved totally geodesic surfaces. Let $M_{K}^{1}$ be the double of $M_{K}^{0}$ along its boundary, i.e. take two copies of $M_{K}^{0}$ and identify their boundaries in the obvious way. We apply Thurston's Uniformization Theorem for Haken manifolds to conclude that $M_{K}^{1}$ is homeomorphic to a closed hyperbolic 3-manifold, and, thus, $\pi_{1}\left(M_{K}^{1}\right)$ is isomorphic to a linear group. This immediately implies that $\pi_{1}\left(M_{K}^{0}\right)$ is also linear.

2.2. Construction of a pseudomanifold $M_{K}$ with large edge links. Let $\Gamma$ be a 4 -colored graph. We will say that $\Gamma$ is $K$-admissible if

a) there are precisely four edges (one of each color) incident to each vertex of $\Gamma$, and

b) every cycle in $\Gamma$ with (alternately) two colors has length $\geq K$.

Lemma 2.1. For every $K$, there are $K$-admissible graphs.

Proof. First, consider the free group $F_{4}$ with four generators $\gamma_{i}, i=1,2,3,4$. It is well known (cf. $\left[\mathrm{St}\right.$ ) that $F_{4}$ is residually finite. Hence there is a homomorphism $\phi$ from $F_{4}$ onto a finite group $G$ so that no nontrivial reduced words of the form $\gamma_{i_{1}}^{k_{1}} \ldots \gamma_{i_{l}}^{k_{l}}, l \in \mathbb{N},\left|k_{1}\right|+\ldots+\left|k_{l}\right| \leq K$, are in $\operatorname{Ker}(\phi)$. Thus, $G$ is a finite group "without short relations (between the generators $\left.\phi\left(\gamma_{i}\right)\right)$ ".

Now construct a graph $\Gamma$ with vertex set $G \times\{1,2\}$, and an edge of color $i$ joining $\left(g_{1}, 1\right)$ to $\left(g_{2}, 2\right)$ whenever $g_{1}=g_{2} \phi\left(\gamma_{i}\right)$. Clearly $\Gamma$ is $K$-admissible since a two-colored cycle in $\Gamma$ determines a relation in the generators $\phi\left(\gamma_{1}\right), \ldots, \phi\left(\gamma_{4}\right)$ of the same length.

Choose $K$ and a $K$-admissible 4-colored graph $\Gamma$, and color each of the four faces of $S$ with a different color. Take a collection of copies of $S$, one for each vertex of $\Gamma$; for each edge $e$ of $\Gamma$, glue the corresponding copies of $S$ along the faces with the same color as $e$. The resulting space $M_{K}$ is a 3-dimensional pseudomanifold, and each edge of $M_{K}$ belongs to at least $K$ copies of $S$.

2.3. Construction of $M_{K}^{0}$. For $i, j=1,2,3,4, i \neq j$, let $A_{i j}$ be a point on the edge $V_{i} V_{j}$ at the distance one from $V_{i}$. For $i=1,2,3,4$ let $S_{i} \subset S$ be the simplex 
with vertices $V_{i}, A_{i j}, j=1,2,3,4, i \neq j$. Let $T$ be the closure of $S \backslash\left(\bigcup_{i=1,2,3,4} S_{i}\right)$, and let $T_{i}=T \cap S_{i}$ be the face of $S_{i}$ opposite $V_{i}$.

Let $M_{K}^{0}$ be the union of the copies of $T$ sitting in $M_{K}$. For any vertex $P_{n}$, $n=1,2, \ldots, m$, of $M_{K}$ let $B_{n}$ denote the union of all copies of $S_{i}$ containing $P_{n}$. Note that $M_{K}^{0}$ is a manifold whose boundary components $\partial B_{n}$ correspond to the vertices $P_{n}$ of $M_{K}, n=1,2, \ldots, m$.

It is easy to see that since $\angle V_{i} A_{i j} A_{i k}<\frac{\pi}{2}$, for all $i \neq j \neq k$, the sets $B_{n}$ are locally convex in $M_{K}$, for all $n=1,2, \ldots, m$. Thus $\partial B_{n}$ is locally convex in $M_{K}^{0}$ for all $n$.

Every $\partial B_{n}$ is a surface glued out of triangles isometric to one of the triangles $T_{i}$. Moreover, the degrees of the vertices in $\partial B_{n}$ are equal to the degrees of corresponding edges in $M_{K}$. In particular, the degrees of all the vertices in $\partial B_{n}$ are greater than $K$. By choosing $K$ large enough we can ensure that $\partial B_{n}$ is nonpositively curved for all $n$ (it is enough to take $K$ bigger than $\frac{2 \pi}{\theta}$ where $\theta$ is the smallest angle of a triangle $T_{i}$ ). The same choice of $K$ ensures that $M_{K}^{0}$ is nonpositively curved at all of its interior points.

We now "double" the space $M_{K}^{0}$ : we consider the space $M_{K}^{1}$ which is the result of gluing together two isometric copies of $M_{K}^{0}$ along their boundary. The boundary components of $M_{K}^{0}$ are locally convex in $M_{K}^{0}$, so we may apply Reshetnyak's theorem (see $[\mathrm{Ba}]$ or $[\mathrm{Re}]$ ) and conclude that $M_{K}^{1}$ has nonpositive curvature.

2.4. $M_{K}^{1}$ satisfies the hypotheses of Thurston's Geometrization Theorem for Haken manifolds when $K$ is sufficiently large. $M_{K}^{1}$ contains an incompressible surface. Every boundary component $\partial B_{n}$ of $M_{K}^{0}$ is an incompressible surface in $M_{K}^{1}$ : the $\partial B_{n}$ are locally convex in $M_{K}^{1}$ and $M_{K}^{1}$ has nonpositive curvature, so the inclusion $\partial B_{n} \rightarrow M_{K}^{1}$ induces a monomorphism of fundamental groups.

$M_{K}^{1}$ is irreducible. By a result of D. Stone ([Sto] $)$, the universal cover $\tilde{M}_{K}^{1}$ is PL-homeomorphic to $R^{3}$, so any embedded PL 2 -sphere in $\tilde{M}_{K}^{1}$ bounds a PL 3-ball. It follows that any embedded PL 2-sphere in $M_{K}^{1}$ bounds a PL 3-ball in $M_{K}^{1}$, so $M_{K}^{1}$ is irreducible.

At this point, we could appeal to $\mathrm{He}$ to conclude that $\pi_{1}\left(M_{K}^{1}\right)$ is a residually finite group. But in fact, we can readily show that $M_{K}^{1}$ admits a hyperbolic structure.

$M_{K}^{1}$ is atoroidal. By the flat torus theorem [Ba], it suffices to show that the universal cover $\tilde{M}_{K}^{1}$ does not contain a 2-flat - a convex subset isometric to the Euclidean plane. Suppose $F \subset \tilde{M}_{K}^{1}$ is a 2-flat. Clearly $F$ must pass through some edge or vertex of $\tilde{M}_{K}^{1}$. If $x \in F$ is an interior point of an edge of $\tilde{M}_{K}^{1}$, then $F$ determines a closed geodesic $\gamma$ of length $2 \pi$ in the space of direction $4 \Sigma_{x}$ at $x$; in fact this implies that $\gamma$ passes through the "poles" of the space of directions, forcing $F$ to contain the entire edge $e$. Consequently, $F$ must contain a vertex $p$ of $\tilde{M}_{K}^{1}$. But then $F$ determines a closed geodesic of length $2 \pi$ in the link of $\tilde{M}_{K}^{1}$ at $p$; but if $K$ is large enough, then no such closed geodesics exist. Hence no such $F \subset \tilde{M}_{K}^{1}$ exists.

2.5. $\pi_{1}\left(M_{K}^{1}\right)$ is residually finite. By Thurston's Geometrization Theorem for Haken manifolds $\mathrm{Th}, \mathrm{Mc}, \mathrm{Ot1}, \mathrm{Ot2}, \mathrm{Ka}, M_{K}^{1}$ admits a hyperbolic metric. Therefore $\pi_{1}\left(M_{K}^{1}\right)$ is isomorphic to a subgroup of the linear group $I \operatorname{som}\left(H^{3}\right)$. Since $M_{K}^{0}$

\footnotetext{
${ }^{4}$ This is just the metric link of $x$ if we view it as a vertex of a suitable subdivision of $\tilde{M}_{K}^{1}$.
} 
is a locally convex subset of $M_{K}^{1}, \pi_{1}\left(M_{K}^{0}\right)$ injects into $\pi_{1}\left(M_{K}^{1}\right)$, and so $\pi_{1}\left(M_{K}^{0}\right)$ is also a linear group. Hence $\pi_{1}\left(M_{K}^{0}\right)$ is residually finite $\mathrm{Ma}$.

2.6. Unwrapping $M_{K}^{0}$ to get a complex whose vertex links contain no homotopically nontrivial closed geodesics shorter than $2 \pi$. Pick $L>2 \pi$. There are only finitely many homotopy classes in each vertex link of $M_{K}$ which can be realized by closed curves of length $\leq L$. These classes determine homotopy classes in the boundary components of $M_{K}^{0}$, and since the boundary components of $M_{K}^{0}$ are incompressible in $M_{K}^{0}$ they determine nontrivial classes $\left\{e_{1}, \ldots, e_{k}\right\}$ in $M_{K}^{0}$. Since $\pi_{1}\left(M_{K}^{0}\right)$ is residually finite there is a finite index normal subgroup $H \triangleleft \pi_{1}\left(M_{K}^{0}\right)$ which does not contain any of the conjugacy classes determined by the $e_{i}$ 's. Let $M^{0}$ be the cover of $M_{K}^{0}$ corresponding to $H$, and let $M \rightarrow M_{K}$ be the corresponding branched cover. The vertex links of $M$ cover vertex links of $M_{K}$ and the covering maps are distance nonincreasing. Due to the choice of $H$, this immediately implies that the vertex links in $M$ contain no homotopically nontrivial closed geodesics of length less than $L$.

2.7. $M$ has nonpositive curvature provided $K$ is large enough. It remains to show that no vertex link $\Sigma$ of $M$ contains homotopically trivial closed geodesics of length less than $2 \pi$. It follows from the methods of $\mathrm{AB}$ that there is a shortest such geodesic $\gamma$ (if there are any at all), and that it is embedded. Let $D \subset \Sigma$ be a disk bounded by $\gamma$.

Let $\partial B$ be the boundary component of $M^{0}$ corresponding to $\Sigma$. Since $\partial B$ is nonpositively curved and there is a biLipschitz homeomorphism $\rho: \partial B \rightarrow \Sigma$ with biLipschitz constant $C=C(S)<\infty$, we conclude (by transferring the isoperimetric inequality for the nonpositively curved disk $\rho^{-1}(D)$ to $\Sigma$ via $\rho$ ) that the area of $D$ is less than $\pi^{3} C^{4}$. By making $K$ large enough we can ensure that every vertex of $\Sigma$ carries integral curvature less than $-\pi^{3} C^{4}$. This implies that $D$ does not contain any vertices.

Now consider the obvious triangulation of $\Sigma$ by spherical triangles. Each edge of this triangulation intersects the interior of the disk $D$ in a finite union of open segments because the geodesic $\gamma$ is "straight" inside each spherical triangle. Consider the collection $W$ of all such open segments. Choose an $I \in W$ so that all other elements of $W$ lie on one side of $I$ in $\operatorname{Int}(D)$. If $A \subset D$ is the component of $D-I$ disjoint from $W$, then the closure of $A$ intersects $\gamma=\partial D$ in a segment which lies in a single triangle $\sigma \subset \Sigma$. This contradicts the fact that $\gamma \cap \sigma$ is straight. Hence when $K$ is large enough $\Sigma$ cannot contain closed geodesics of length less than $2 \pi$. Thus, $M$ has large links and consequently (see $\mathrm{Gr}$ or $[\mathrm{GH}]$ for the proof) has nonpositive curvature.

\section{REFERENCES}

[AB] S. Alexander, R. Bishop. The Hadamard-Cartan theorem in locally convex metric spaces. Enseign. Math, (2) 36 (1990), no. 3-4, 309-320. MR 92c:53044

[Ba] W.Ballmann. Lectures on spaces of nonpositive curvature. With an appendix by Misha Brin. DMV Seminar, 25. Birkhauser Verlag, Basel, 1995. MR 97a:53053

[BFK1] D.Burago, S.Ferleger, A.Kononenko. Uniform estimates on the number of collisions in semi-dispersing billiards. Annals of Mathematics, (2) 147 (1998), 695-708. MR 99f:58120

[BFK2] D.Burago, S.Ferleger, A.Kononenko. Topological entropy of semi-dispersing billiards. Ergodic Theory and Dynamical Systems, 18 (1998), 791-805. MR 99m:58146 
[BFK3] D.Burago, S.Ferleger, A.Kononenko. Unfoldings and global bounds on the number of collisions for generalized semi-dispersing billiards. Asian J. Math., 2 (1998), 141-152. MR 2000c:37039

[GH] E. Ghys, P. de la Harpe (eds.). Sur les groupes hyperboliques d'aprés Mikhael Gromov. Prog. Math., vol. 83, Birkhäuser, Boston, 1990. MR 92f:53050

[Gr] M.Gromov. "Hyperbolic groups." in Essays in group theory, S.M.Gersten (ed.). M.S.R.I. Publ., Vol.8: 75-263, Springer 1987. MR 89e:20070

[He] J.Hempel. Residual finiteness for 3-manifolds. Combinatorial group theory and topology, 379-396, Ann. of Math. Stud., 111, Princeton Univ. Press, Princeton, NJ, 1987. MR 89b:57002

[Ka] M. Kapovich. Hyperbolic manifolds and Discrete Groups. Notes on Thurston's Hyperbolization. University of Utah Lecture Notes, 1995. To appear in Birkhäuser, Progress in Mathematics.

[Ma] A.Malcev. On isomorphic matrix representations of infinite groups. (Russian) Rec. Math. [Mat.Sbornik] N.S., 8, no. 50, 405-422, 1940. MR 2:216d

[Mc] C.McMullen. Iteration on Teichmuller space. Invent. Math. 99 (1990), no. 2, 425-454. MR 91a:57008

[Ot1] J.-P. Otal. Le théorème d'hyperbolisation pour les variétès fibrées de dimension 3. Astérisque 235, Société mathématique de France, 1996. MR 97e:57013

[Ot2] J.-P. Otal. Thurston's hyperbolization of Haken manifolds. Surveys in differential geometry, Vol. III (Cambridge, MA, 1996), Int. Press, Boston, MA, 1998, pp. 77-194. MR 2000b:57025

[Re] Yu.G.Reshetnyak (ed.). Geometry 4, non-regular Riemannian geometry. Encyclopedia of Mathematical Sciences, Vol.70, 1993. MR 94i:53038

[Sp] E. Spanier. Algebraic Topology. Springer, 1996. MR 96a:55001

[St] J.Stillwell. Classical Topology and Combinatorial Group Theory. Springer-Verlag, New York, 1993. MR 94a:57001

[Sto] D.Stone. Geodesics in piecewise linear manifolds. Trans. Amer. Math. Soc., 215 (1976), 1-44. MR 53:6464

[Th] W.Thurston. Three-dimensional manifolds, Kleinian groups and hyperbolic geometry. Bull. Amer. Math. Soc. (N.S.), 6, no. 3, 357-381, 1982. MR 83h:57019

Department of Mathematics, The Pennsylvania State University, University Park, Pennsylvania 16802

E-mail address: burago@math.psu.edu

Renaissance Technology Corporation, 600 Rt. 25-A, East Setanket, New York 11787

Department of Mathematics, University of Utah, Salt Lake City, Utah 84112-0090

E-mail address: bkleiner@math.utah.edu

Renaissance Technology Corporation, 600 Rt. 25-A, East Setanket, New York 11787

E-mail address: kononena@yahoo.com 